\title{
Gastrostomía endoscópica percutánea en ancianos: indicaciones, seguridad y desenlaces
}

\section{Percutaneous Endoscopic Gastrostomy in elderly, indications, safety and outcomes}

\author{
Diana Patricia Atencio ${ }^{1}$, Ángela Gabriela Blanco Pérez¹, William Otero Regino, MD. ${ }^{2}$
}

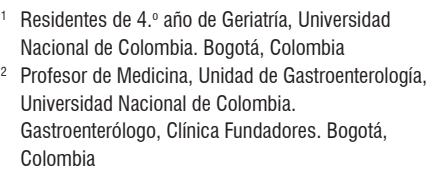

1 Residentes de $4 .{ }^{\circ}$ año de Geriatría, Universidad Nacional de Colombia. Bogotá, Colombia

2 Profesor de Medicina, Unidad de Gastroenterología, Universidad Nacional de Colombia. Gastroenterólogo, Clínica Fundadores. Bogotá, Colombia

Fecha recibido: $23-07-14$ Fecha aceptado: $02-02-15$

\begin{abstract}
Resumen
Introducción: la gastrostomía endoscópica percutánea (GEP) se ejecuta cada vez con mayor frecuencia en ancianos con alteraciones deglutorias, sin importar su condición física, mental o funcional. En nuestro medio se desconocen sus complicaciones y desenlaces. Objetivo: el propósito del estudio es determinar las indicaciones, tasa de complicaciones y desenlaces a corto y largo plazo en el seguimiento de hasta de 5 años. Pacientes y métodos: estudio descriptivo retrospectivo de pacientes mayores de 60 años de edad hospitalizados con indicación de gastrostomía endoscópica percutánea y atendidos en la Clínica Fundadores en el período comprendido entre enero de 2008 y junio del 2013; fueron excluidos pacientes menores de 60 años, realización de gastrostomía abierta o GEP fallida. Se realizó revisión de historias clínicas y seguimiento telefónico de los pacientes. Resultados: de los 135 pacientes con indicación de GEP, 96 fueron incluidos, media de edad 77,5 \pm 9 años, 38 hombres $(39,5 \%)$. La indicación para la GEP fue la incapacidad para la deglución por evento cerebrovascular $(32,89 \%)$, demencia $(30,26 \%)$ u otras causas $(36,85 \%)$. La hipertensión arterial fue la comorbilidad más frecuente. La complicación más común asociada con la GEP fueron los síntomas gastrointestinales (32,9\%). No hubo mortalidad asociada con el procedimiento. Conclusiones: la GEP es segura para el aporte enteral a los pacientes ancianos, sin embargo, no demostró beneficio en aquellos pacientes asociados con demencia. Es importante un manejo interdisciplinario previo para definir la alimentación por gastrostomía en ancianos.
\end{abstract}

\section{Palabras clave}

Anciano, gastrostomía endoscópica percutánea, complicaciones.

\begin{abstract}
Introduction: Percutaneous endoscopic gastrostomies (PEG) are performed more and more frequently in elderly patients suffering from problems with swallowing. The procedure is used regardless of patients' physical, mental and functional status, but little is known about complications and outcomes here in Colombia. Objective: The purpose of the study is to determine indications for performance of PEG, rate of complications, and outcomes in short and long term follow-ups (five years). Patients and methods: This is a retrospective and descriptive study of patients over the age of 60 who were hospitalized for percutaneous endoscopic gastrostomy at the Clínica Fundadores between January 2008 and June 2013. Patients younger than 60 years of age, those who had open gastrostomies, and those whose PEG procedure failed were excluded. Patients medical records were reviewed and telephone follow-ups of patients were conducted. Results: Of the 135 patients with indications for PEG, 96 patients were included. The mean age was $77.5 \pm 9$ years, and 38 of the patients were men $(39.5 \%)$. The reasons for performance of PEG were inability to swallow due to a cerebrovascular event $(32.89 \%)$, dementia $(30.26 \%)$ and other causes (36.85). Hypertension was the most common comorbidity. The most common complications associated with PEG were gastrointestinal symptoms (32.9\%). There was no mortality associated with the procedure. Conclusions: PEG is a safe way for elderly patients to receive enteral tube feeding (ETF), but we found no benefit for patients with dementia. Interdisciplinary management prior to the procedure is important to determine whether or not ETF should be used for elderly patients.
\end{abstract}

\section{Keywords}

Elderly, percutaneous endoscopic gastrostomy, complications. 


\section{INTRODUCCIÓN}

En la actualidad es una realidad el aumento cada vez mayor de la población anciana. Se estima que para el 2050 a nivel mundial la población mayor de 60 años de edad se habrá duplicado y pasará de $11 \%$ en 2006 a 22\%, y en América 1 de cada 5 individuos será mayor de 65 años (1); en Colombia las personas mayores de 60 años representan el 10,5\% de la población total (2). Con el aumento de la expectativa de vida no solo incrementan las enfermedades crónicas sino también los problemas nutricionales, la carga sociofamiliar y el uso de recursos económicos $(1,2)$. En éste grupo etario es mayor el riesgo de desnutrición, fundamentalmente por la limitación en la ingesta por diversas morbilidades, polifarmacia, problemas de dentición, discapacidad, depresión, aislamiento y pobreza (3). En los pacientes con demencia las alteraciones nutricionales ocurren hasta en un $86 \%$ (4). La valoración y el soporte nutricional del anciano hacen parte de su manejo integral $(3,4)$.

A nivel mundial, la gastrostomía endoscópica percutánea (GEP), desde su descripción inicial en 1980 (5), ha tenido gran impacto por su seguridad, sencillez y eficacia para proveer alimentación cuando existen alteraciones de la deglución en personas con un sistema digestivo indemne y que se prevé que su trastorno será superior a 1 mes $(6,7)$. Se incluyen en este grupo pacientes con enfermedades terminales o con fases avanzadas de enfermedades neurológicas crónicas, aunque hay poca evidencia de calidad que respalde su utilización $(8,9)$. Skelly y colaboradores (10) encontraron que el número de gastrostomías realizadas en los Estados Unidos aumentó de 61000 en 1988 a 121000 en 1995, y en el Reino Unido la nutrición enteral domiciliaria presentó un incremento de 6,5\% en el período 2004-2005 y el 82\% corresponde a adultos con GEP (11). En 2005, según el reporte BANS (11), más del 65\% de las GEP se realizaron a mayores de 60 años y cerca de $45 \%$ a mayores de 70 años. En la actualidad no contamos con datos en Latinoamérica.

Las indicaciones de GEP están dadas por la disminución de la ingesta debido a procesos neurológicos que producen disfagia neuromotora (12-14) en demencia, accidente cerebrovascular (ACV), esclerosis lateral amiotrófica, esclerosis múltiple, enfermedad de Parkinson, entre otras, y también se indica en enfermedades tumorales de la cavidad orofaríngea, el cuello y el esófago (15). Hay otras indicaciones menos frecuentes tales como descompresión del tracto digestivo alto en carcinomas avanzados, quemaduras extensas, fibrosis quística, sida avanzado o traumatismos graves $(12,16)$. Antes de realizar la GEP es ideal que se identifiquen los pacientes que realmente puedan beneficiarse del procedimiento (10), lo cual incluye que tengan esperanza de vida superior a 30 días (9). Hay evidencia de su beneficio en pacientes con secuelas de $\mathrm{ACV}$ y neoplasias de cabeza y cuello que estén en tratamiento con radioterapia y/o quimioterapia $(14,15,17)$. Por el contrario, en pacientes con demencia avanzada y disfagia o enfermedades rápidamente progresivas, el beneficio de la GEP es cuestionable $(13,18)$ debido a que no hay evidencia de que mejore la supervivencia, calidad de vida, estado nutricional y que pueda disminuir el riesgo de broncoaspiración o de aparición de úlceras por presión (13).

La GEP se considera un procedimiento seguro, con baja tasa de morbilidad (3\%-12\%) y mortalidad (0\%-2\%) (16, $17,19,20)$. Entre las complicaciones mayores que ocurren en el 3\% de los pacientes se encuentra la fascitis necrotizante, el síndrome de "buried bumper" (21), la peritonitis, la perforación gastroesofágica y la fístula gastrocólica o colocutánea, y entre las complicaciones menores, las cuales son tardías y más frecuentes, están la infección local y/o celulitis, fuga de contenido gástrico, sangrado, neumoperitoneo, obstrucción o desplazamiento de la sonda, úlcera gástrica y molestias gastrointestinales al iniciar la alimentación tales como vómito, diarrea o estreñimiento $(10,19,20)$. Si bien la nutrición enteral se plantea como una medida terapéutica, en la actualidad existe controversia respecto a si hace parte del soporte básico en pacientes con enfermedades terminales $(22,23)$. El médico no familiarizado con las intervenciones al final de la vida toma decisiones que en muchos casos pueden ir en contra de una mejor calidad de vida $(22,23)$ y por esto es necesario un equipo interdisciplinario para el manejo de estos pacientes, donde los especialistas en geriatría y gastroenterología se deben convertir en figuras clave a la hora de definir estas conductas.

$\mathrm{Al}$ tener en cuenta que en nuestro medio la GEP es un procedimiento disponible y ofertado por la mayoría de los hospitales y que no hay estudios sobre la evolución a largo plazo en pacientes ancianos, se decidió realizar el presente trabajo con el fin de determinar las indicaciones de su solicitud, la tasa de complicaciones y los desenlaces a corto plazo en las personas de 60 o más a quiénes se les realizó el procedimiento.

\section{PACIENTES Y MÉTODOS}

Estudio realizado en la Unidad de Gastroenterología y Endoscopia Digestiva de la Clínica Fundadores durante el período entre enero de 2008 a junio de 2013. La población de estudio incluyó a los pacientes mayores de 60 años que se les realizó GEP. Fueron excluidos pacientes menores de 60 años, o mayores de 60 años a quienes se realizó gastrostomía por cirugía, GEP fallida o cuando los datos de la historia clínica estaban incompletos. De forma retrospectiva se revisaron las historias clínicas y se estableció contacto telefónico con los pacientes o cuidadores, aplicándose un cuestionario específico para obtención de datos de quie- 
nes cumplían los criterios de inclusión. La información se tabuló en Excel y se analizaron los datos clínicos para verificar su validez.

\section{TÉCNICA GASTROSTOMÍA ENDOSCÓPICA PERCUTÁNEA}

La GEP se realizó en la forma usual en la sala de endoscopia utilizando el kit de gastrostomía 20Fr Kimberly, que en Colombia tiene un costo de $\$ 780000$ pesos colombianos. La técnica utilizada ha sido descrita previamente $(7,24)$. La GEP se realizó sin sedación en el 90\% de los pacientes.

\section{ANÁLISIS ESTADÍSTICO}

Se describió la población de estudio a través de elementos de la estadística descriptiva para las variables sociodemográficas.

Para realizar las comparaciones entre grupos y estimar las relaciones presentadas en el análisis descriptivo se utilizó el método de regresión logística como herramienta econométrica. En algunos casos los grupos fueron comparados usando un test estadístico de medias tipo $t$, el cual compara la media de la variable de interés de ambos grupos y establece si la diferencia entre ellas es estadísticamente diferente de 0 . El modelo logit es comúnmente utilizado para predecir la probabilidad de un evento cuya ocurrencia depende de otros factores cuando el evento y la mayoría de factores estudiados son medidos con variables dicotómicas -variables que solo toman 2 valores ( 0 y 1$)$ - como es el caso de este estudio.

Los coeficientes estimados para cada factor o variable independiente fueron convertidos en odds ratios (OR) para ser interpretados; estos se leen como el cambio proporcional que se produce en la probabilidad de ocurrencia del evento estudiado por cada unidad de cambio de la variable independiente o factor en cuestión.

\section{CONSIDERACIONES ÉTICAS}

Según la resolución $\mathrm{N}^{\circ} 8430$ de 1993 se establece que durante el presente estudio no se realizaron intervenciones que pusieran en riesgo la vida de los pacientes, recolectando los datos a través de la revisión de historias clínicas, informes endoscópicos del procedimiento y llamadas telefónicas. Por lo tanto, este estudio se clasifica como "investigación sin riesgo" y no requiere consentimiento informado por parte del paciente.

\section{RESULTADOS}

Durante el período del estudio se solicitaron 135 GEP de las que se realizaron 100, en la Unidad de Gastroenterología y Endoscopia Digestiva de la Clínica Fundadores, de la ciudad de Bogotá, y se logró el seguimiento a 96 pacientes (figura 1). De estos 96 pacientes, 38 eran hombres (39,5\%). La edad promedio de la población de estudio fue 77,5 \pm 9 años (rango 60-94 años) (tabla 1).

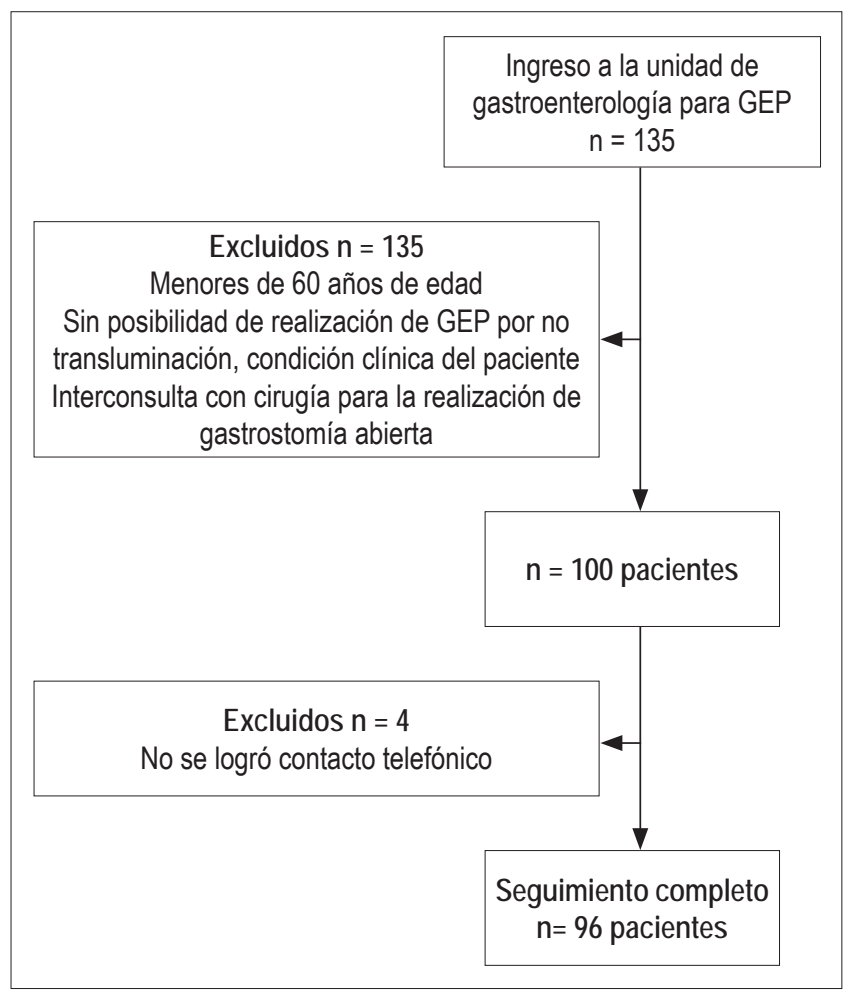

Figura 1. Población y seguimiento.

Las indicaciones para el procedimiento y las comorbilidades de los pacientes incluidos se muestran en la tabla 1.

De la población del estudio, 5 pacientes $(5,26 \%)$ tenían traqueostomía al momento de la GEP y 5 pacientes recibían anticoagulación, la cual fue suspendida antes del procedimiento. El 27,6\% de los pacientes tenía personal asistencial de salud a cargo de la gastrostomía, 5,26\% procedían de ancianatos y/o unidad de cuidados crónicos y el 1,32\% tenía diagnóstico de "sospecha de abandono social" (tabla 1).

Del total de pacientes incluidos, $66(68,42 \%)$ requirieron nueva hospitalización por causas médicas sin relación directa con la parte técnica de la ejecución del procedimiento. Dentro de los diagnósticos de reingreso los más relevantes fueron infección de vías urinarias, neumonía aspirativa, declinación funcional, dificultad respiratoria, entre otros. La tabla 2 muestra la frecuencia con la que ocurren los diagnósticos presentados.

El $41,67 \%$ del total de los pacientes $(n=40)$ requirió al menos una hospitalización posterior a la GEP. El 32,89\% $(\mathrm{n}=31)$ tuvo síntomas gastrointestinales como diarrea y distensión abdominal; el 10,53\% $(\mathrm{n}=10)$ de los pacientes 
Tabla 1. Características de la población del estudio.

\begin{tabular}{|c|c|}
\hline Característica & Total, $\mathrm{n}(\%)$ \\
\hline Edad (media $\pm D E)$, años & $77,5 \pm 9$ (rango $60-94$ ) \\
\hline \multicolumn{2}{|l|}{ Género } \\
\hline Femenino & $58(60,5)$ \\
\hline Masculino & $38(39,5)$ \\
\hline \multicolumn{2}{|l|}{ Comorbilidades } \\
\hline Hipertensión arterial & $59(61,8)$ \\
\hline ACV & $54(56,58)$ \\
\hline Dependencia funcional & $44(46)$ \\
\hline Demencia no especificada & $36(36,84)$ \\
\hline Broncoaspiración & $24(25)$ \\
\hline EPOC & $19(19,74)$ \\
\hline Alteración del estado de conciencia & $16(17,11)$ \\
\hline Sarcopenia & $16(17,11)$ \\
\hline Enfermedad neoplásica & $14(14,47)$ \\
\hline Falla cardiaca & $13(13,16)$ \\
\hline Epilepsia & $11(11,84)$ \\
\hline Diabetes mellitus & $10(10,53)$ \\
\hline Incontinencia urinaria & $10(10,53)$ \\
\hline Hipotiroidismo & $10(10,53)$ \\
\hline Dislipidemia & $10(10,53)$ \\
\hline Úlceras por presión & $9(9,21)$ \\
\hline Anemia & $8(7,89)$ \\
\hline Hipertensión pulmonar & $8(7,89)$ \\
\hline Enfermedad renal crónica & $6(6,58)$ \\
\hline TVP/TEP & $5(5,26)$ \\
\hline Fibrilación auricular & $5(5,26)$ \\
\hline Uso de anticoagulación & $5(5,26)$ \\
\hline Trauma craneoencefálico & $5(5,26)$ \\
\hline Traqueostomía & $5(5,26)$ \\
\hline Asma & $4(3,95)$ \\
\hline Obesidad & $4(3,95)$ \\
\hline Enfermedad de Parkinson & $4(3,95)$ \\
\hline Fractura y/o reemplazo de cadera & $4(3,95)$ \\
\hline SAHOS & $3(2,63)$ \\
\hline HVDA & $3(2,63)$ \\
\hline Artritis reumatoide & $1(1,32)$ \\
\hline Otros & $47(48,68)$ \\
\hline \multicolumn{2}{|c|}{ Indicación para realización de gastrostomía: trastorno deglutorio } \\
\hline Asociado con ACV & $32(32,89)$ \\
\hline Asociado con demencia & $29(30,26)$ \\
\hline Asociado con otras causas & $35(36,85)$ \\
\hline \multicolumn{2}{|l|}{ Características sociales de la población } \\
\hline $\begin{array}{l}\text { Cuidador con entrenamiento en manejo de } \\
\text { gastrostomía }\end{array}$ & $26(27,6)$ \\
\hline Institucionalización en hogares geriátricos & $5(5,26)$ \\
\hline Sospecha de abandono social & $1(1,32)$ \\
\hline
\end{tabular}

ACV: accidente cerebrovascular; EPOC: enfermedad pulmonar obstructiva crónica; HVDA: hemorragia de vías digestivas altas; SAHOS, síndrome de apnea hipopnea obstructiva del sueño; TVP/ TEP: trombosis venosa profunda/tromboembolismo pulmonar. presentó infección local superficial en el sitio de la GEP que no ameritó retirarla; el 9,6\% ( $\mathrm{n}=9)$ síndrome de "buried bumper"; hemorragia leve en el sitio de la gastrostomía $3,85 \%(\mathrm{n}=4)$ que se controló inyectando adrenalina. $\mathrm{Al}$ $23,9 \%(n=23)$ de los 96 pacientes se les realizó cambio de sonda de gastrostomía: $12,5 \%(\mathrm{n}=12)$ por desalojo, $7,3 \%$ $(\mathrm{n}=7)$ por deterioro de la misma y $4,1 \%(\mathrm{n}=4)$ por ruptura del balón cuando la sonda era de recambio (tabla 3 ).

Tabla 2. Diagnósticos de reingreso posterior a la GEP.

\begin{tabular}{lc}
\hline \multicolumn{1}{c}{ Diagnóstico o patología } & Total, $\mathbf{n}(\%)$ \\
\hline Infección de vías urinarias & $15(15,4)$ \\
Neumonía aspirativa & $13(13,5)$ \\
Declinación funcional & $7(7,7)$ \\
Dificultad respiratoria & $7(7,7)$ \\
Sepsis & $4(3,8)$ \\
Traqueostomía & $2(1,9)$ \\
Alteración hidroelectrolíica & $2(1,9)$ \\
Úlceras por presión & $2(1,9)$ \\
Derivación ventrículo peritoneal & $2(1,9)$ \\
\hline
\end{tabular}

Tabla 3. Complicaciones asociadas con la GEP.

\begin{tabular}{lc}
\hline & Total, $\mathbf{n}(\%)$ \\
\hline Mayor & \\
Síndrome de buried bumper & $9(9,6)$ \\
Laparotomía por trayecto fistuloso & $1(1,32)$ \\
Menor & \\
Síntomas gastrointestinales: diarrea, distensión & $31(32,89)$ \\
Infección del estoma & $10(10,53)$ \\
Sangrado por ostomía & $4(3,85)$ \\
Cambio GEP & $23(23,9)$ \\
Desplazamiento/desalojo sonda & $12(12,5)$ \\
Deterioro de sonda & $7(7,3)$ \\
Ruptura de balón & $4(4,1)$ \\
\hline
\end{tabular}

$\mathrm{Al} 64,58 \%(\mathrm{n}=62)$ de los pacientes incluidos se les administró el aporte enteral con dieta hecha en casa y el 35,6\% $(\mathrm{n}=34)$ recibían suplemento nutricional de fórmula. Al $83 \%(\mathrm{n}=80)$ se les administró medicamentos a través de la sonda de gastrostomía. En el $25 \%(\mathrm{n}=24)$ de la población del estudio se documentó percepción, por parte del paciente o del cuidador, de mejoría en la condición clínica posterior a la GEP y solo el 10,42\% ( $\mathrm{n}=10)$ del total de los pacientes presentó mejoría del patrón de deglución durante el seguimiento (tabla 4).

El 38,16\% ( $\mathrm{n}=37)$ de los 96 pacientes estudiados murió luego de la realización de la GEP, dentro de este grupo $16,22 \%(n=6)$ muere entre el día 0 y 5 posterior a la reali- 
zación de la GEP, el 21,62\% ( $\mathrm{n}=8)$ muere entre el día 11 y 30 , y el $62,16 \%(n=23)$ muere en un tiempo mayor a 30 días, con un promedio de 68,7 días, sin documentarse en la historia clínica o en la entrevista telefónica que la causa de muerte estuviera en relación directa con el procedimiento (tabla 5).

Tabla 4. Aporte por GEP, percepción de la mejoría y recuperación del patrón de deglución.

\begin{tabular}{lc}
\hline \multicolumn{1}{c}{ Aporte enteral } & Total, $\mathbf{n}(\%)$ \\
\hline Dieta artesanal & $62(64,58)$ \\
Fórmula suplementaria & $34(35,41)$ \\
Aporte de medicamentos & $80(83)$ \\
Percepción de mejoría clínica & $24(25)$ \\
Recuperación de la deglución & $10(10,42)$ \\
\hline
\end{tabular}

Tabla 5. Temporalidad del deceso posterior a la GEP.

\begin{tabular}{cc}
\hline Tiempo de muerte en días & Total, $\mathbf{n}(\%)$ \\
\hline $0-5$ & $6(16,22)$ \\
$6-10$ & $0(0)$ \\
$11-30$ & $8(21,62)$ \\
$31-89$ & $11(29,73)$ \\
$\geq 90$ & $12(32,43)$ \\
\hline
\end{tabular}

En la figura 2 se observa mayor supervivencia cuando la GEP se realizó en pacientes con EV, comparados con los que tenían demencia.

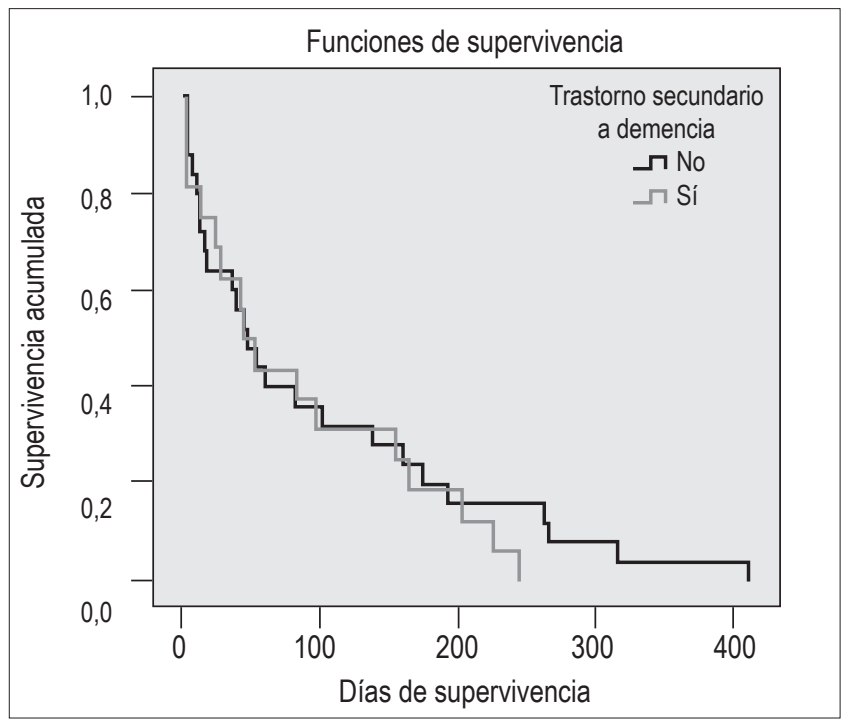

Figura 2. Curva de supervivencia Kaplan-Meir.

$\mathrm{Al}$ realizar un análisis de regresión logística se encontró que la probabilidad de morir después de la GEP es 3 veces mayor para aquellos pacientes cuya indicación para el procedimiento fue trastorno de deglución asociado con demencia (diferencia que es significativa al 5\%). Lo anterior se refleja al documentar que el 51,7\% $(n=15)$ de los pacientes a los cuales se les hizo GEP con la indicación de trastorno deglutorio asociado con demencia murió después del procedimiento. De ellos, 4 fallecieron en los 5 días siguientes al procedimiento $(26,6 \%)$, mientras que para los pacientes cuya indicación para GEP era trastorno de deglución asociado con ACV u otras causas, el porcentaje de muerte fue $32,8 \%(n=22)$ y 2 de ellos $(9,52 \%)$ murieron en los siguientes 5 días (tabla 6).

Tabla 6. Comparación de la mortalidad de acuerdo con indicación de la GEP y supervivencia.

\begin{tabular}{lccc}
\hline $\begin{array}{c}\text { Indicación } \\
\text { GEP }\end{array}$ & $\begin{array}{c}\text { Total, } \mathbf{n} \\
(\%)\end{array}$ & $\begin{array}{c}\text { Muerte a } 5 \text { días } \\
\text { Total, } \mathbf{n}(\%)\end{array}$ & $\begin{array}{c}\text { Supervivencia (>5 días) } \\
\text { Total, } \mathbf{n}(\%)\end{array}$ \\
\hline Demencia & $15(51,7)$ & $4(26,6)$ & $11(73)$ \\
$\begin{array}{l}\text { ACV y } \\
\text { otras }\end{array}$ & $22(32,8)$ & $2(9,52)$ & $20(91)$ \\
\hline
\end{tabular}

Ninguno de los pacientes con indicación de trastorno deglutorio asociado con demencia presentó mejoría en el proceso de deglución después de la realización de la GEP, mientras que el $15 \%(n=10)$ de los sujetos que se sometieron a este procedimiento por trastorno de deglución asociado con ACV u otras causas sí lo hicieron (diferencia significativa al 5\%) (tabla 7).

Tabla 7. Comparación de recuperación de la deglución de acuerdo con la indicación de la GEP.

\begin{tabular}{lc}
\hline \multicolumn{1}{c}{ Indicación de GEP } & Total, $\mathbf{n}(\%)$ \\
\hline Demencia & $0(0)$ \\
ACV y otras causas & $10(15)$ \\
\hline
\end{tabular}

De los 31 pacientes que presentaron síntomas gastrointestinales posteriores al inicio del aporte enteral por esta vía, el 59\% $(\mathrm{n}=18)$ correspondían al grupo con indicación de GEP asociada con demencia y $41 \%(n=13)$ fueron los pacientes con trastorno deglutorio asociado con otras causas (tabla 8).

\section{DISCUSIÓN}

El actual es el estudio más grande hasta la fecha que evalúa los resultados clínicos posteriores a la realización de la GEP en pacientes ancianos en Colombia. Las indicaciones para la realización de la GEP en este trabajo fueron clasificadas en 3 grupos: trastorno de deglución asociado con ACV agudo (32,88\%), demencia (30,26\%) y otras causas $(47,37 \%)$ que incluyeron cáncer de cabeza y cuello, trauma 
craneoencefálico, fístula traqueoesofágica, secuelas de encefalopatía hipóxico isquémica por paro cardiorrespiratorio, compromiso de deglución por enfermedades del sistema nervioso central; éstas son similares a otros estudios internacionales, incluido un estudio latinoamericano realizado en Perú $(20,25,26)$. Dependiendo de la edad de los pacientes varía la frecuencia de las indicaciones, siendo muy frecuente en los ancianos el trastorno de la deglución asociado con demencia $(26,27)$.

Tabla 8. Comparación de síntomas gastrointestinales: diarrea, distensión abdominal de acuerdo con la indicación de la GEP.

\begin{tabular}{lc}
\hline \multicolumn{1}{c}{ Indicación de GEP } & Total, $\mathbf{n}(\%)$ \\
\hline Demencia & $18(59)$ \\
ACV y otras causas & $13(41)$ \\
\hline
\end{tabular}

En el presente estudio se encontró que las comorbilidades más frecuentes en la población anciana a la cual se le realizó GEP fueron HTA $(61,8 \%)$, ataque cerebrovascular agudo (56,58\%), dependencia funcional (46\%), demencia $(36,84 \%)$ y al menos 1 episodio de broncoaspiración (25\%), lo cual concuerda con los resultados de otros autores $(20,29)$. En el $26 \%$ de los pacientes se produjeron complicaciones directamente relacionadas con la GEP durante el seguimiento. El 10,53\% presentó infección leve del estoma que no ameritó el retiro de la sonda; esta complicación en las diferentes series varía entre 6,9\% (19) a 31,2\% $(7,16,25)$. Otra complicación menor fue hemorragia en el sitio de inserción de la GEP en el 3,85\%, la cual se controló en todos los pacientes con inyección de adrenalina; esta complicación es similar a la encontrada en otra serie (20). La única complicación mayor encontrada en esta cohorte fue el síndrome de "buried bumper", el cual ocurrió en casi el $10 \%$ de los pacientes, siendo superior a lo encontrado por otros autores $(30,31)$; esta complicación usualmente es causada por la inadecuada manipulación de la sonda por parte de los cuidadores o por tracción accidental por parte del paciente (21) y por lo tanto su incidencia varía dependiendo de la población, del seguimiento endoscópico de los pacientes, y de las tasas de mortalidad de los mismos según la enfermedad de base $(21,31)$. Globalmente, en el $41,7 \%$ de los pacientes se produjeron complicaciones relacionadas con la GEP, siendo superior a la encontrada en otras series $(17,20,29)$, aunque el manejo intrahospitalario de las mismas fue inferior a 2 días. Las complicaciones gastrointestinales fueron las más frecuentes, 32,89\% de los casos, representadas por diarreas y distensión abdominal, y éstas probablemente tienen relación con el tipo de nutrición suministrada. En este trabajo, este tipo específico de alteraciones fue inferior al $40 \%$ encontrado en un trabajo similar (19). En el presente estudio ningún paciente tuvo eventos adversos cardiorrespiratorios entre el ingreso a la sala del procedimiento y su salida, como tampoco se encontraron complicaciones mayores tales como abscesos, hemorragias severas, peritonitis, broncoaspiración masiva o neumotórax, que han sido descritas en otros trabajos; tampoco hubo mortalidad directamente relacionada con el procedimiento como ha sido descrito en otras investigaciones $(8,17,25,29,32)$.

El 23,9\% de los pacientes del estudio necesitó cambio de la sonda de gastrostomía: $12,5 \%$ por desalojo, $7,3 \%$ por deterioro del tubo, y 4,1\% por ruptura del balón; estas son consideradas como complicaciones menores de tipo mecánico (19). En la serie española del Hospital de Alicante (19) se reportaron complicaciones de pérdida de la sonda en 1 ocasión, en el 10,3\% de los casos, y más de una vez en el $1,7 \%$. Gundogan y colaboradores reportaron que el $12 \%$ de los pacientes requirió cambio de sonda debido a malfuncionamiento, desalojo y extravasación del contenido gástrico (20) y Chicharro encontró que en el $25 \%$ de los pacientes se produjo pérdida accidental de la sonda (17). Las diferencias en los datos anteriores probablemente se pueden explicar porque en este estudio y en el estudio español (17) había un importante número de pacientes con demencia, los cuales frecuentemente tienen alteraciones del comportamiento que pueden provocar extracción traumática de la sonda (33-35). En el presente estudio el $38,16 \%$ de los pacientes murió después de la realización de la GEP; dentro de este grupo, 17,24\% falleció entre el día 0 y el día 5 , el $20,69 \%$ entre el día 11 y el día 30 , y el $62,12 \%$ en un tiempo mayor a 30 días después de la realización del procedimiento. Si bien esta mortalidad es mayor que la encontrada en la mayoría de las series (36-39), es similar a la Gundogan (20) y la explicación más probable es el mayor número de pacientes con demencia en estado avanzado o terminal.

En la curva de Kaplan-Meier se encontró que la supervivencia de los pacientes es menor cuando la GEP se realizó por trastorno de la deglución asociado con demencia en comparación con otras alteraciones. Al tener en cuenta que aproximadamente el $40 \%$ de los pacientes falleció dentro del primer mes después de la GEP y que la mayoría de los pacientes con ese desenlace tenían demencia, sería recomendable que la decisión de este procedimiento la tome una junta multidisciplinaria ya que el "kit" de GEP tiene un alto costo en Colombia. La pertinencia de la GEP en pacientes con demencia avanzada debería evaluar los potenciales y controvertidos beneficios del procedimiento (23) con la alta probabilidad de muerte por esta entidad y más aún cuando en otros estudios no se han demostrado beneficios claros en pacientes con esta condición $(13,43)$. Fisiopatológicamente la nutrición enteral aumenta las secreciones gástricas, la incontinencia fecal y urinaria, y las 
úlceras por presión (43). Algunos autores consideran que en estos pacientes lo único que hace la GEP y el soporte nutricional es prolongar el proceso de muerte del paciente (44), otros consideran que la decisión de colocar GEP en un paciente con demencia debe tomarse con consideraciones éticas (45-49), y sociedades científicas como la ESPEN no recomiendan este tipo de nutrición en las personas con demencia severa (50).

Al igual que en otras series $(19,20)$, la deglución espontánea se recuperó en el 10,53\% de los pacientes y la sonda fue retirada con éxito, sin embargo esto no sucedió en ninguno de los pacientes con demencia avanzada. Este estudio tiene limitaciones como ser retrospectivo y haberse realizado en una única institución, lo cual implica que sus resultados no pueden generalizarse.

Con base en los resultados encontrados se concluye lo siguiente: 1. La GEP en pacientes adultos mayores es un procedimiento técnicamente seguro, fácil de realizar pero con potenciales complicaciones durante el seguimiento a largo plazo, y 2. No se encontró beneficio cuando la indicación fue trastorno de deglución asociada con demencia.

\section{Financiación}

Los costos del presente trabajo fueron asumidos en su totalidad por los autores.

\section{Conflicto de intereses}

Ninguno por declarar.

\section{Agradecimientos}

A la Unidad de Gastroenterología, Endoscopia Digestiva, Enfermedades del Hígado de la Clínica Fundadores por permitirnos el acceso a los reportes de las gastrostomías e historias clínicas para la ejecución del presente trabajo.

Especial agradecimiento a Andrea Atencio de León por la organización de la bases de datos y el análisis estadístico.

A Liliana Oino, asistente de Gastroenterología y estudiante de Ingeniaría Biomédica por la diligencia y prontitud para la disponibilidad de las historias clínicas y de la base de datos de los pacientes del presente estudio.

\section{REFERENCIAS}

1. United Nations, Department of Economic and Social Affairs, Population Division. World Population Ageing 2013.

2. DANE (Departamento Administrativo Nacional de Estadística). Estimación y proyección nacional, departamental y municipal total por área 1985-2020. Disponible en: http//www.dane.gov.co, consultado en enero de 2014.
3. John BK, Bullock M, Brenner L, McGaw C, Scolapio J. Nutrition in the elderly. Frequently asked question. Am J Gastroenterol 2013;108:1252-66.

4. Mitchell SL, Teno JM, Kiely DK, Shaffer ML, Jones RN, Prigerson HG, et al. The clinical course of advanced dementia. N Engl J Med 2009;361:1529-1538.

5. Gauderer MWL, Ponsky Jl, Izant J. Gastrostomy without laparotomy, a percutaneous technique. J Pedriatr Surg $1980 ; 15: 872-5$.

6. Volkert D, Berner YN, Berry E, Cederholm T, Coti BP, Milne A. et al. ESPEN (European Society for Parenteral and Enteral Nutrition). Guidelines on Enteral Nutrition: Geriatrics. Clin Nutr 2006;25:330-60.

7. Sebastian JJ. Gastrostomía endoscópica percutánea. Técnica e indicaciones. Endocrinol Nutr. 2004;51:158-62.

8. Pereira JL, Belda O, Parejo J, Serrano P, Bozada JM, Fraile J, et al. La gastrostomía endoscópica percutánea. Realidad en la práctica nutricional clínica intra y extrahospitalaria. Rev Clin Esp 2005;205:472-7.

9. Razavi F, Gross S, Katz S. Endoscopy in the elderly: Risks, benefits, and yield of common endoscopic procedures. Clin Geriatr Med 2014;30:133-47.

10. Skelly R. Are we using percutaneous endoscopic gastrostomy appropriately in the elderly? Curr Opin Clin Nutr Metab Care 2002;5:35-42.

11. Jones B, Holden C, Dalzell M, Micklewright A, Glencorse C. Annual BANS Report Artificial Nutrition Support in the UK 2005. A Report by the British Artificial Nutrition Survey (BANS), a committee of BAPEN (The British Association for Parenteral and Enteral Nutrition) UK 2005:13-17.

12. Slater R. Percutaneous endoscopic gastrostomy feeding: Indications and management. Brit J Nur 2009;18:1036-43.

13. Sampson EL, Candy B, Jones L. Enteral tube feeding for older people with advanced dementia. Cochrane Database of Systematic Reviews. 2009;2:1-25.

14. Britton JE, Lipscomb G, Mohr PD, Rees WD, Young AC. The use of percutaneous endoscopic gastrostomy (PEG) feeding tubes in patients with neurological disease. J Neurol 1997;244:431-4.

15. Saunders J, Brown MS, Hirata RM, Jaques DA. Percutaneous endoscopic gastrostomy in patients with head and neck malignancies. Am J Surg. 1991;162:391-93.

16. Erdogan A. Single endoscopist-performed percutaneous endoscopic gastrostomy tube placement. World J Gastroenterol 2013;19:4172-6.

17. Chicharro L. Complicaciones inmediatas de la gastrostomía percutánea de alimentación: 10 años de experiencia. Nutr Hosp 2009;24:73-6.

18. Gillick MR. Rethinking the role of tube feeding in patients with advanced dementia. N Engl J Med 2000;342:206-10.

19. Wanden-Berghe C, Muñoz J, Cantó C, Domenech MD, Reyes MD, Pérez Moya C. Gastrostomía Endoscópica Percutánea (PEG): 10 años de experiencia. Nutr Hosp 2010;25:949-53.

20. Gundogan K, Yurci A, Coskun R, Baskol R, Gursoy S, Hebbar G, et al. Outcomes of percutaneous endoscopic gas- 
trostomy in hospitalized patients at a tertiary care center in Turkey. Eur J Clin Nutr 2014;68:437-40.

21. Moreno N, Otero W, Gómez M. Síndrome de "buried bumper” (botón interno de la gastrostomía enterrado): desenterrando la solución. Rev Col Gastroenterol 2007;22:51-7.

22. Cardin F. Special considerations for endoscopists on PEG indications in older patients. ISRN ISRN Gastroenterol 2012;2012:60714.

23. Peñaloza A, Suárez J, Blanco L, Peñaloza A. Gastrostomía endoscópica percutánea: ¿Es éticamente aceptable? Rev Col Gastroenterol 2013;28:150-60.

24. Chang WK, Hsieh TY. Safety of percutaneous endoscopic gastrostomy in high-risk patients. J Gastroenterol Hepatol 2013;28(Suppl 4):118-22.

25. Yriberry S, Monge V, Cabrera F, Barriga E, Vesco E. Gastrostomía endoscópica percutánea: experiencia prospectiva de un centro privado nacional. Rev Gastroenterol Perú 2004;24:314-22.

26. Elia M, Russell CA, Stratton RJ, Shaffer J, Micklewright A, Wood S, et al. Trends in artificial nutritional support in the UK during 1996-2000. A report by the British Artificial Nutrition Survey (BANS). British Association of Parenteral and Enteral Nutrition. Maidenhead, UK: BAPEN 2001.

27. Mendiratta P, Tilford JM, Prodhan P, Curseen K, Azhar G, Wei JY. Trends in percutaneous endoscopic gastrostomy placement in the elderly from 1993 to 2003. Am J Alzheimers Dis Other Demen 2012;27:609-13.

28. Malmgren A, Hede G, Karlström B, Cederholm T, Lundquist $\mathrm{P}$, Wirén $\mathrm{M}$, et al. Indications for percutaneous endoscopic gastrostomy and survival in old adults. FoodNutr Res 2011;55:6037-42.

29. McClave SA, Chang WK. Complications of enteral access. Gastrointest. Endosc 2003;58:739-51.

30. Finocchiaro C, Galleta R, Rovera G, Ferrari A, Todros L, Vuolo A, et al. Percutaneous endoscopic gastrostomy a long term follow-up. Nutrition 1997;13:520-3.

31. Meine G, Lukashok H, Mello G, Mansur G, Guimarães D, Carvalho R, et al. Buried Bumper Syndrome as a complication of percutaneous endoscopic gastrostomy in cancer patients: The brazilian experience. Digest Endosc 2007; 19:22-5.

32. Schrag S, Sharma R, Jaik N, Seamon M, LukaszczykJ, Martin $\mathrm{N}$, et al. Complications related to percutaneous endoscopic gastrostomy (PEG) tubes. A comprehensive clinical review. J Gastrointestin Liver Dis 2007;16:407-18.

33. Warren J, Rohrer J. Frontotemporal dementia. BMJ 2013;347:4827-35.

34. Ahronheim JC, Mulvihill M, Sieger C, Park P, Fries BE. State practice variations in the use of tube feeding for nursing home residents with severe cognitive impairment. J Am Geriatr Soc 2001;49:148-52.

35. Mitchell SL, Teno JM, Roy J, Kabumoto G, Mor V. Clinical and organizational factors associated with feeding tube use among nursing home residents with advanced cognitive impairment. JAMA 2003;290:73-80.

36. López L, Iñiguez F, Santos E, Balado M, Pérez-Carnero A. Gastrostomía percutánea endoscópica. Experiencia en un hospital general. Rev Esp Enferm Dig 1994;85:173-6.

37. Martín A, Espinós J, Forné M, Rius J, Corbera G, Quintana $S$, et al. Gastrostomía endoscópica percutánea: estudio de 35 enfermos. Med Clin 1994;103:449-51.

38. Park R, Allison M, Lang J, Spence E, Morris A, Danesh B, et al. Randomised comparison of percutaneous endoscopic gastrostomy and nasogastric tube feeding in patients with persisting neurological dysphagia. BMJ 1992;304:1406-9.

39. Gencosmanoglu R, Koc D, Tozun N. Percutaneous endoscopic gastrostomy: results of 115 cases. Hepatogastroenterology 2003;50:886-8.

40. Kaw M, Sekas G. Long-term follow-up of consequences of percutaneous endoscopic gastrostomy (PEG) tubes in nursing home patients. Dig Dis Sci 1994;39:738-43.

41. Callahan C, Haag K, Weinberger M, Tierney WM, Buchanan NN, Stump TE, et al. Outcomes of percutaneous endoscopic gastrostomy among older adults in a community setting. J Am Geriatr Soc 2000;48:1048-54.

42. Mccann R, Hall W, Groth-Juncker A. Comfort care for terminally ill patients. The appropriate use of nutrition and hydration. JAMA 1994;272:1263-6.

43. Candy B, Sampson E, Jones L. Enteral tube feeding in older people with advanced dementia: Findings from a Cochcrane systematic review. Int J Palliative Nurs 2009;55:396-404.

44. Morgenstern L, Laquer M, Treyzon L. Ethical challenges of percutaneous endoscopic gastrostomy. Surg Endosc 2005; 19:398-400.

45. Sanders D, Carter M, D’Silva J, James G, Bolton R, Bardhan $\mathrm{K}$. Survival analysis in percutaneous endoscopic gastrostomy feeding: A worse outcome in patients with dementia. Am J Gastroenterol 2000;95:1472-5.

46. Nair S, Hertan H, Pitchumoni CS. Hypoalbuminemia is a poor predictor of survival after percutaneous endoscopic gastrostomy in elderly patients with dementia. Am J Gastroenterol 2000;95:133-6.

47. Mitchell SL, Kiely DK, Lipsitz LA. The risk factors and impact of survival of feeding tube placement in nursing home residents with severe cognitive impairment. Arch Intern Med 1997; 157:327-32.

48. Murphy LM, Lipman TO. Percutaneous endoscopic gastrostomy does not prolong survival in patients with dementia. Arch Intern Med 2003;163:1351-3.

49. Dharmarajan TS, Unnikrishnan D, Pitchumoni CS. Percutaneous endoscopic gastrostomy and outcome in dementia. Am J Gastroenterol. 2001;96:2556-2563.

50. Körner U, Bondolfi A, Bühler E, MacFie J, Meguid M, Messing B, et al. Ethical and legal aspects of enteral nutrition. ESPEN guidelines. Clin Nutr 2006;25:196-202. 